\title{
An efficient terahertz detector based on an optical hybrid cavity
}

\section{Thomas Siday, Robert J. Thompson, Samuel Glass, Ting-Shan Luk, John L. Reno, et al.}

Thomas Siday, Robert J. Thompson, Samuel Glass, Ting-Shan Luk, John L. Reno, Igal Brener, Oleg Mitrofanov, "An efficient terahertz detector based on an optical hybrid cavity," Proc. SPIE 10531, Terahertz, RF, Millimeter, and Submillimeter-Wave Technology and Applications XI, 1053109 (23 February 2018); doi: $10.1117 / 12.2290971$

SPIE. Event: SPIE OPTO, 2018, San Francisco, California, United States 


\title{
An efficient terahertz detector based on an optical hybrid cavity
}

\author{
Thomas Siday*a, Robert J Thompson ${ }^{\mathrm{a}, \mathrm{b}}$ Samuel Glass ${ }^{\mathrm{a}}$, Ting-Shan Luk ${ }^{\mathrm{c}, \mathrm{d}}$, John L Reno ${ }^{\mathrm{c}, \mathrm{d}}$, Igal \\ Brener $^{\mathrm{c}, \mathrm{d}}$ and Oleg Mitrofanov ${ }^{\mathrm{a}, \mathrm{c}}$ \\ ${ }^{a}$ Electronic and Electrical Engineering, University College London, London, UK; ${ }^{b}$ London Center \\ for Nanotechnology, University College London, London, $\mathrm{WC} 1 \mathrm{H} 0 \mathrm{AH} ;{ }^{\mathrm{c}} \mathrm{Center}$ for Integrated \\ Technologies, Sandia National Laboratory, Albuquerque, NM 87185, USA; ${ }^{\mathrm{d}}$ Sandia National \\ Laboratory, Albuquerque, NM 87185, USA
}

\begin{abstract}
We demonstrate an efficient terahertz (THz) detector based on an optical hybrid cavity, which consists of an optically thin photoconductive layer between a distributed Bragg reflector (DBR) and an array of electrically isolated nanoantennas. Using a combination of numerical simulations and optical experiments, we find a hybrid cavity design which absorbs $>75 \%$ of incident light with a $50 \mathrm{~nm}$ photoconductive layer. By integrating this optical hybrid cavity design into a THz detector, we see enhanced detection sensitivity at the operation wavelength $(\sim 815 \mathrm{~nm})$ over designs which do not include the nanoantenna array.
\end{abstract}

Keywords: Terahertz detectors, photonic nanostructures, optical cavity

\section{INTRODUCTION}

A significant technological challenge in the terahertz $(\mathrm{THz})$ frequency range is the development of efficient nanoscale $\mathrm{THz}$ detectors. The efficiency of photoconductive (PC) THz detectors based on low temperature grown (LT) GaAs is limited by the bulk material properties: the mean free path of photo-excited carriers, and the optical absorption length. A popular method to mitigate the material limitations is to modify the photoconductive antenna to incorporate plasmonic nano-electrodes ${ }^{1-6}$. These electrodes support an optical plasmon resonance, which strongly localizes the generation of electron-hole pairs to the vicinity of the nano-electrodes. This modification means that when a $\mathrm{THz}$ field is present, significantly more carriers can reach an electrode before recombining within the semiconductor. However, the close spacing of the electrically connected nano-electrodes may lead to increased dark noise.

One alternative is to use a photonic structure to trap light within an optically thin semiconductor layer, a method utilized previously for photovoltaics ${ }^{7-10}$. A structure for THz detection, known as an optical hybrid cavity is shown in Fig. 1(a) ${ }^{11}$. Here, an optically thin photoconductive layer of LT-GaAs is sandwiched between a DBR and a Gold nanoantenna array, which is electrically isolated from the photoconductive layer with a $15 \mathrm{~nm}$ thick layer of $\mathrm{Al}_{2} \mathrm{O}_{3}$. This prevents the nanoantennas interacting with the electrical properties of the device. Details of the fabrication process can be found in ${ }^{12}$. The operation of the device is as follows: infra-red (IR) light $(\lambda=800 \mathrm{~nm})$ excites a dipolar resonance in the nanoantennas, which scatter the light into the photoconductive layer. Photons which escape the photoconductive layer are reflected back by the DBR, forming a cavity mode similar to an optical microcavity within the photoconductive layer ${ }^{11}$. The DBR was designed using 5 pairs of alternating $60 \mathrm{~nm} \mathrm{Al} \mathrm{A}_{0: 55} \mathrm{Ga}_{0: 45} \mathrm{As}(n=3.25$ at $800 \mathrm{~nm})$ and $55 \mathrm{~nm}$ GaAs $(n=2.68$ at $800 \mathrm{~nm})$ layers, resulting in a stop band centered near $810 \mathrm{~nm}$.

*tom.siday.15@ucl.ac.uk

Terahertz, RF, Millimeter, and Submillimeter-Wave Technology and Applications XI,

edited by Laurence P. Sadwick, Tianxin Yang, Proc. of SPIE Vol. 10531, 1053109

(C) 2018 SPIE · CCC code: $0277-786 X / 18 / \$ 18 \cdot$ doi: $10.1117 / 12.2290971$ 
In this work, we vary several critical parameters of the hybrid cavity design: The thickness of the photoconductive layer $L_{G a A s}$, the periodicity of the nanoantenna array $a$ and $b$, and the size the individual nanoantennas $L_{a}$ and $L_{b}$. These parameters are shown in Fig. 1(b).

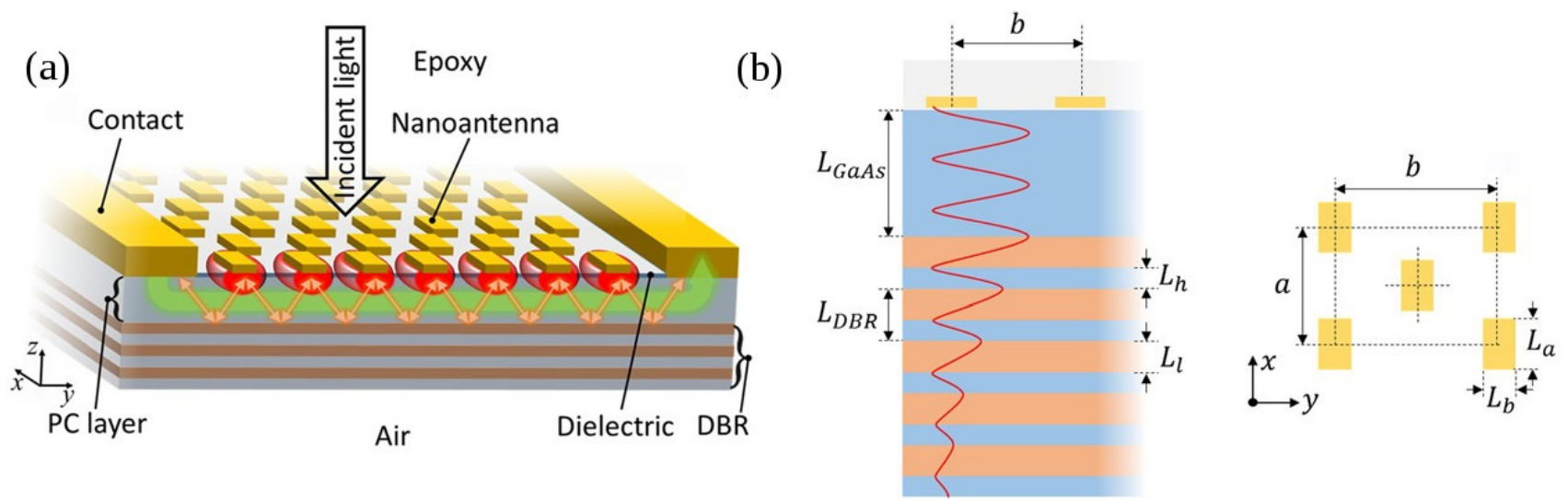

Figure 1. (a) Visualization of the optical hybrid cavity. (b) Cross-section of the cavity, showing the critical parameters of the device. The red line represents the optical field within the cavity ${ }^{12}$

Figure 2 shows optical reflectivity and transmission spectra for the hybrid cavity, where (a) are numerical spectra calculated using the finite-difference time-domain (FDTD) method $^{13}$, and (b) are experimental reflectivity spectra. Transmission $(T)$ and reflectivity (R) spectra are evaluated throughout this chapter to ease comparison between experimental and numerical measurements. Where device absorption is required, this is approximated as $1-(R+T)$. As can be seen Fig. 2, without the nanoantennas, the structure behaves identically to a DBR, with a stop-band centered at $800 \mathrm{~nm}$ (black dashed line). When the nanoantenna array is introduced, the reflectivity (solid black line) reduces to $<10 \%$ at $\sim 810 \mathrm{~nm}$, whilst transmission through the device remains low. This reflectivity suppression clearly indicates the presence of the hybrid cavity mode discussed above.

\section{— Hybrid Cavity Array Reflectivity — Hybrid Cavity Array Transmission - - - DBR Reflectivity - - - DBR Transmission}
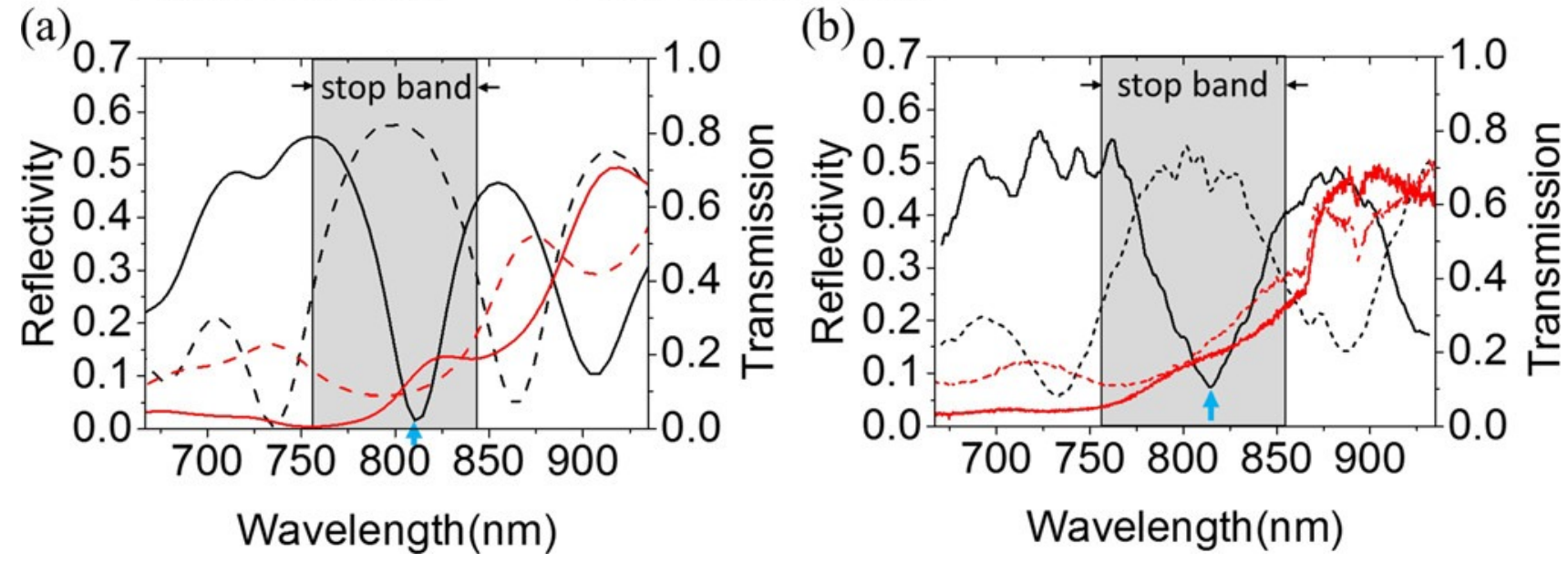

Figure 2. Spectra showing in black the reflectivity, and in red the transmission for the hybrid cavity (solid lines). The dashed lines show the same results when no nanoantenna array is present. The results in (a) are simulated using the FDTD method, and (b) are experimental measurements. The hybrid cavity parameters are $\mathrm{a}=250 \mathrm{~nm}, \mathrm{~b}=350 \mathrm{~nm}$ and $\mathrm{LGaAs}=270 \mathrm{~nm}^{12}$. 


\section{THICKNESS OF THE PHOTOCONDUCTIVE LAYER}

We first consider the photoconductive layer thickness, $L_{G a A s}$. Figure 3 shows reflectivity (a) and absorption spectra (b) for varying $L_{G a A s}$ thickness. Three zones of high absorption are visible, with maxima between 800 and $810 \mathrm{~nm}$ for $L_{\text {GaAs }}=$ 270,160 and $50 \mathrm{~nm}$. This effect can be understood by considering the field distribution of the cavity mode, depicted in Fig. 1(b). When $L_{\text {GaAs }}$ is varied, the wavelength of the mode supported by the hybrid cavity also varies. The resonance within the hybrid cavity can be approximated with the $k$-vector condition:

$$
k=\frac{2 \pi}{\lambda}=\frac{m_{r} \pi}{L_{G a A s} n_{G a A s}+m_{D B R} L_{D B R} n_{D B R}}
$$

Here, $m_{r}$ is a half integer multiple representing the mode order of resonance in the cavity, $n_{\text {GaAs }}$ is the refractive index of the photoconductive layer, $m_{D B R}$ is the number of DBR pairs of thickness $L_{D B R}$, and $n_{D B R}$ is the averaged refractive index of the DBR. This $k$-vector condition is plotted in Fig. 3(b) for $m_{r}=5.5,6.5$, and 7.5. Within the DBR stopband (750$875 \mathrm{~nm}$ ), this $k$-vector condition coincides with the absorption peak, confirming the hybrid cavity mode is excited. From this $k$-vector condition, it is possible to express the optimum thickness of the hybrid cavity: $L_{G a A s}=\left(\frac{1}{4}+\frac{1}{2} N\right) \lambda / n_{G a A s}$, where $N=0,1,2 \ldots$
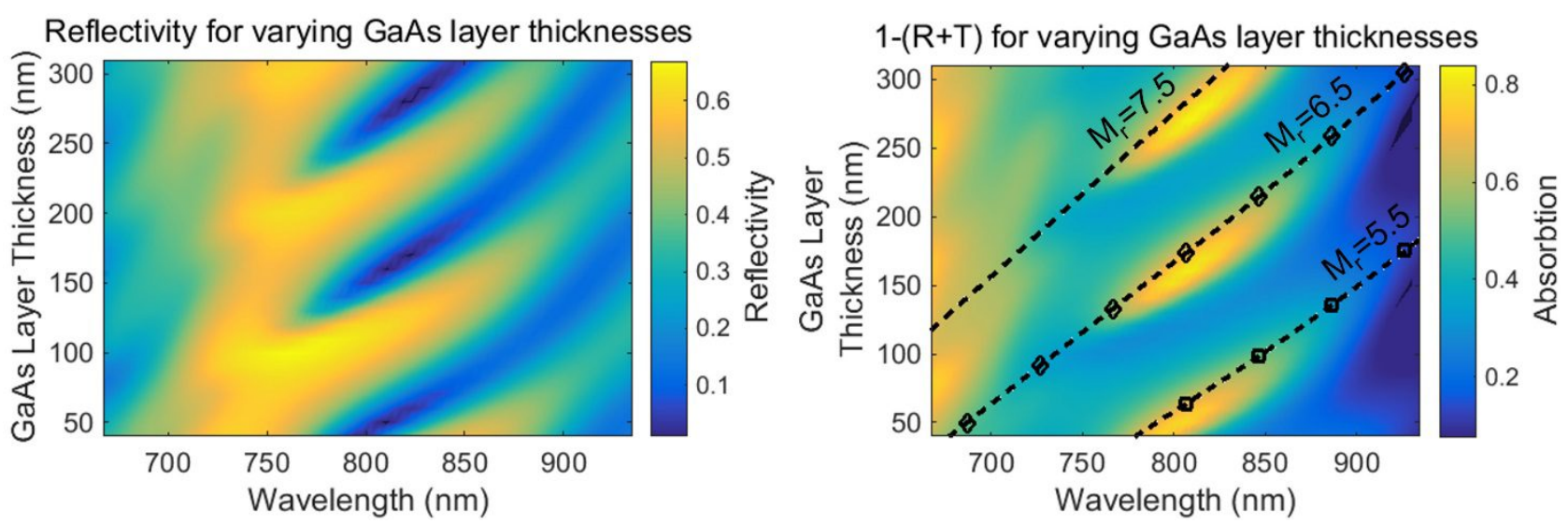

Figure 3. Normalized reflectivity (a) and absorption (b) when the photoconductive layer thicknesses is varied ${ }^{12}$.

\section{PERIODICITY OF THE NANOANTENNA ARRAY}

The second parameter investigated is the periodicity of the nanoantenna array $a$ and $b$ (Fig. 1(b)), as it was suggested ${ }^{11}$ that the periodicity could affect the coupling of light into the hybrid cavity. A number of hybrid cavity structures were fabricated to investigate this. Figure 4(a) shows scanning electron microscope (SEM) images of nanoantenna arrays with periodicities $b=350 \mathrm{~nm}$ and $450 \mathrm{~nm}(a=250 \mathrm{~nm})$. Figure 4(b) and (c) show numerically calculated absorption spectra for varying periodicity $b$ and $a$, respectively. What is interesting here is there is practically no change in the position or maximum absorption of the hybrid cavity mode for a wide range of periodicities. Only at large periodicities, larger than the effective wavelength $\left(\lambda=n_{\text {GaAs }}\right)$ does the spectrum significantly change. This behavior is similar to that seen in the context of photovoltaics by Munday et al. ${ }^{14}$

Figure 4(d) and (e) show the wavelength and peak absorption for the cavity mode respectively for different nanoantenna array periodicities. For smaller $k$-vector (large periodicities) the center wavelength of the mode shifts significantly, which indicates the presence of multiple modes. For larger $k$, where $k_{x}^{2}+k_{y}^{2}>k_{0}^{2} n^{2}$, the mode is significantly more stable, and shifts by only $\sim 15 \mathrm{~nm}$ across the range of periodicities simulated. Therefore, the range of periodicities where the hybrid cavity mode is supported can be approximately expressed as

$$
\left(\frac{2 \pi}{a}\right)^{2}+\left(\frac{2 \pi}{b}\right)^{2}=k_{0}^{2} n^{2}
$$

Figure 4(f) shows experimental reflectivity measurements confirming the stability of the hybrid cavity mode across a range of periodicities. 
(a)

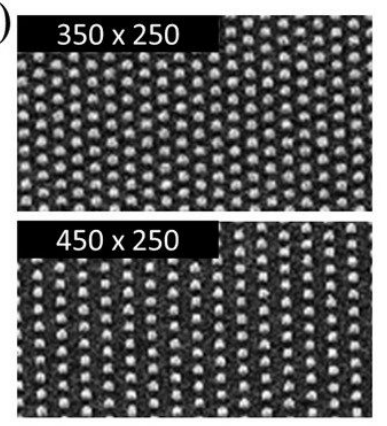

(b)

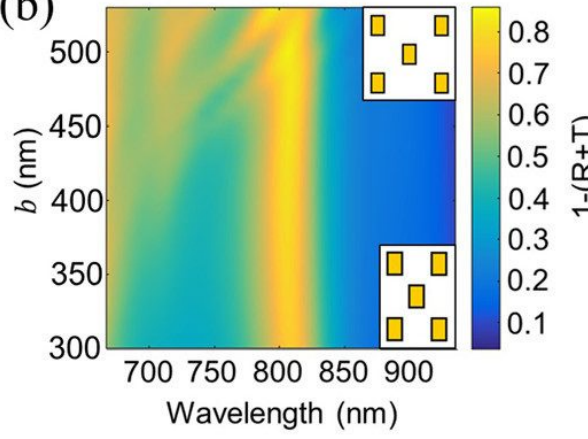

(c)

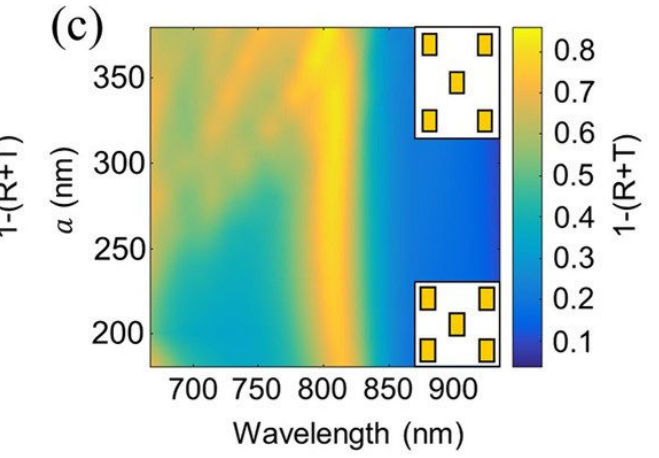

(e)

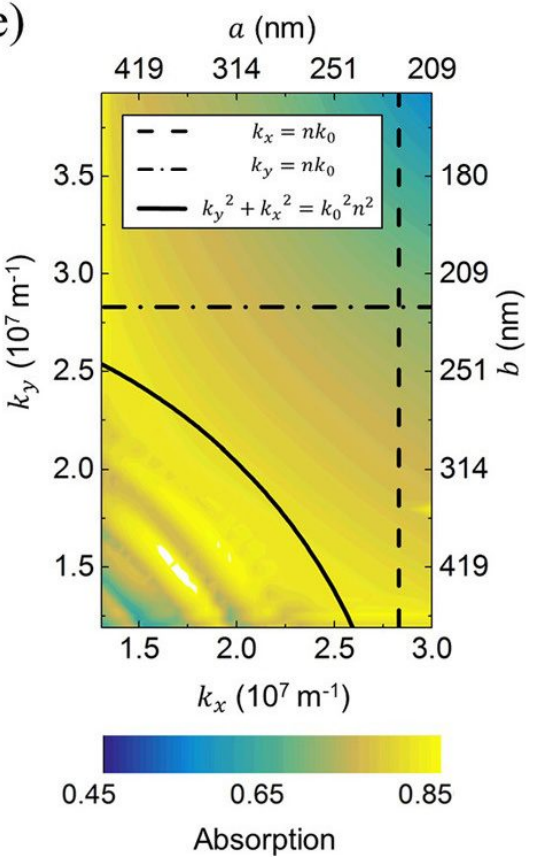

(f)

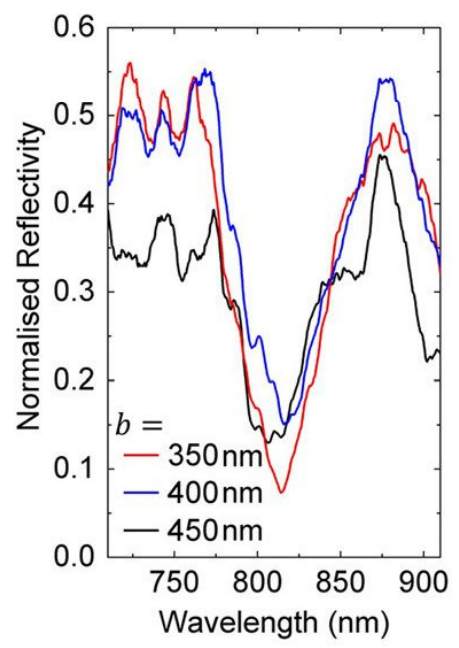

(d)

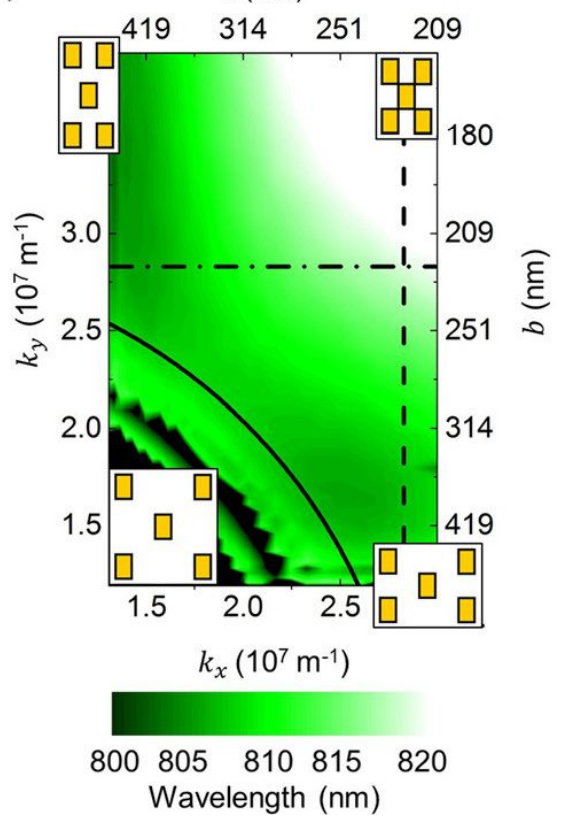

Figure 4. The effect of nanoantenna periodicity on optical absorption within the hybrid cavity. (a) SEM images of hybrid cavities with different nanoantenna periodicity. Here $L_{G a A s}=270 \mathrm{~nm}$, and the electron beam current density is $500 \mu \mathrm{C} / \mathrm{cm}^{2}$. (b) And (c) show optical absorption spectra as the periodicity in $b$ and $a$ is varied, respectively. (d) Wavelength of maximum absorption and (e) the normalized peak absorption when the periodicity is varied for both $a$ and $b$. (f) experimental measurements for different nanoantenna periodicities. ${ }^{12}$

\section{SIZE OF THE NANOANTENNAS}

The final parameter of interest in optimizing the hybrid cavity structure is the size of the individual nanoantennas. Figure 5(a) shows numerically calculated reflectivity spectra for nanoantenna lengths $L_{a}=70-110 \mathrm{~nm}$. The location of minimum reflectivity is seen to shift to longer wavelengths for increasing nanoantenna length, as would be expected for a dipole antenna ${ }^{15}$. Experimental verification was provided by altering the electron beam dosage during fabrication, which has the effect of altering the nanoantenna size, and can be seen in the SEM in Fig. 5(b) for electron beam doses $450 \mu \mathrm{C} / \mathrm{cm}^{2}$ and $650 \mu \mathrm{C}=\mathrm{cm}^{2}$.

Figure 5(c) shows experimental reflectivity measurements for the electron beam doses shown in the SEM images, plus $550 \mu \mathrm{C} / \mathrm{cm}^{2}$. This effect of nanoantenna size can be explained intuitively by considering the phase of light reflected from the hybrid cavity. We can consider light reflected from the hybrid cavity consist of two components: reflections form the nanoantenna array, and reflection from the DBR. While the phase of light reflected by the DBR remains approximately constant across the stopband, the phase of light reflected by the nanoantennas shifts significantly across the nanoantenna resonance. Therefore, by altering the length $\left(L_{a}\right)$ of the nanoantennas, their resonance can be tuned such that light 
reflected from the nanoantennas destructively interferes with light reflected from the DBR. For the hybrid cavity design where $L_{\text {GaAs }}=250 \mathrm{~nm}, a=340 \mathrm{~nm}$ and $b=250 \mathrm{~nm}$, this occurs most efficiently when $L_{a}=90 \mathrm{~nm}$.

(a)

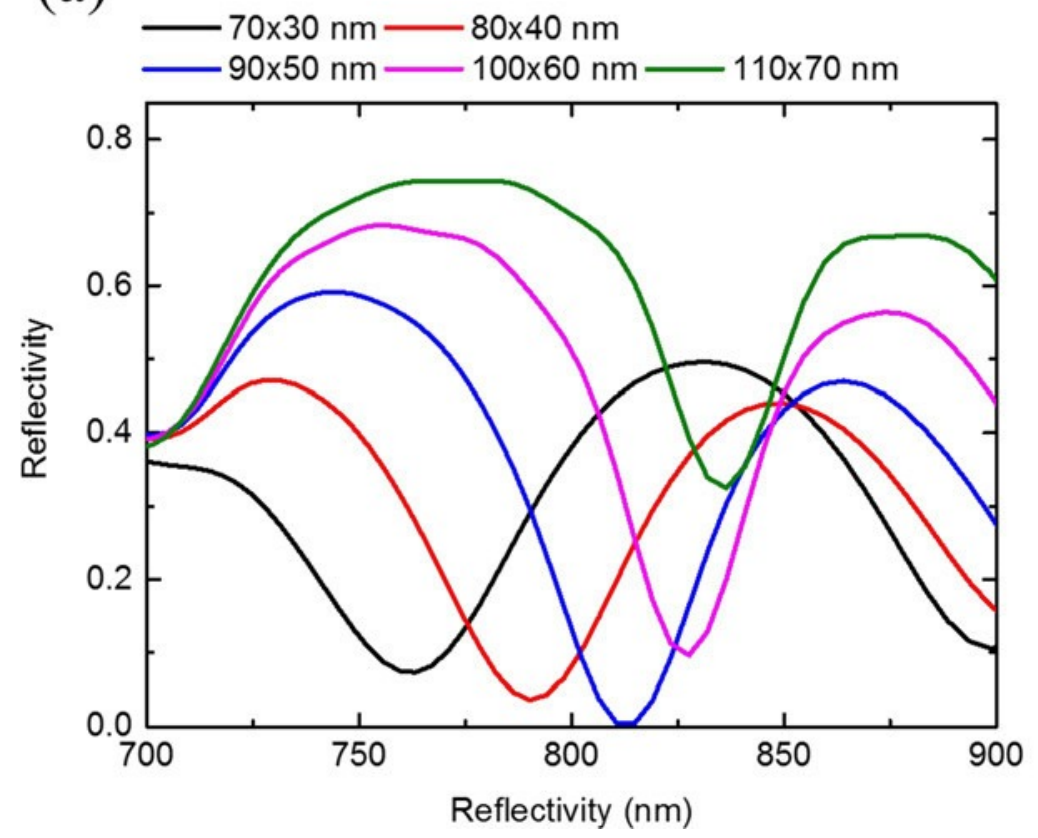

(b)

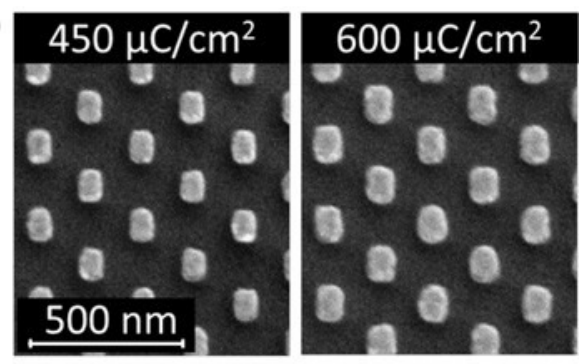

(c)

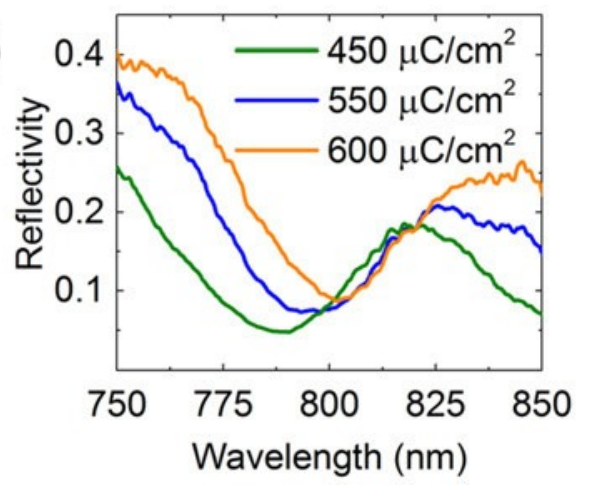

Figure 5. (a) Simulated reflectivity spectra for different nanoantenna sizes $L_{a}$ and $L_{b}$. (b) SEM images showing nanoantenna arrays with different electron beam exposure. (c) Reflectivity spectra of the cavities displayed in (b) ${ }^{12}$.

\section{TERAHERTZ CHARACTERISATION}

To evaluate the performance of the hybrid cavity design for $\mathrm{THz}$ detection, the hybrid cavity design was integrated into a photoconductive $\mathrm{THz}$ detector. For comparison, an identical photoconductive antenna was fabricated without the nanoantenna array. Figure 6(a) shows $\mathrm{THz}$ waveforms, generated with a $\mathrm{ZnTe}$ crystal in a $\mathrm{THz}$ time-domain spectroscopy (TDS) system (the experimental configuration is inset), and measured with (red) and without (black) the nanoantenna array. The IR pulse from the Ti:Sapphire laser is tuned to $815 \mathrm{~nm}$. In both cases the shape and spectrum (Fig. 6(a) and 6(c)) of the pulse is the same, with the peak photocurrent higher when the nanoantenna array is present.

Figure 6(b) shows the performance of the hybrid cavity $\mathrm{THz}$ detector when the wavelength of the IR gate pulse is varied. In this experiment, a THz detector consisting of a $1 \mu \mathrm{m}$ thick layer of InAs on a $30 \mu \mathrm{m}$ layer of GaAs replaces the ZnTe crystal to prevent the $\mathrm{THz}$ beam becoming misaligned as the wavelength of the IR gate pulse is varied. Relative to the detector without the nanoantenna array, the sensitivity of the hybrid cavity detector is enhanced by $17 \%$ at $815 \mathrm{~nm}$. 

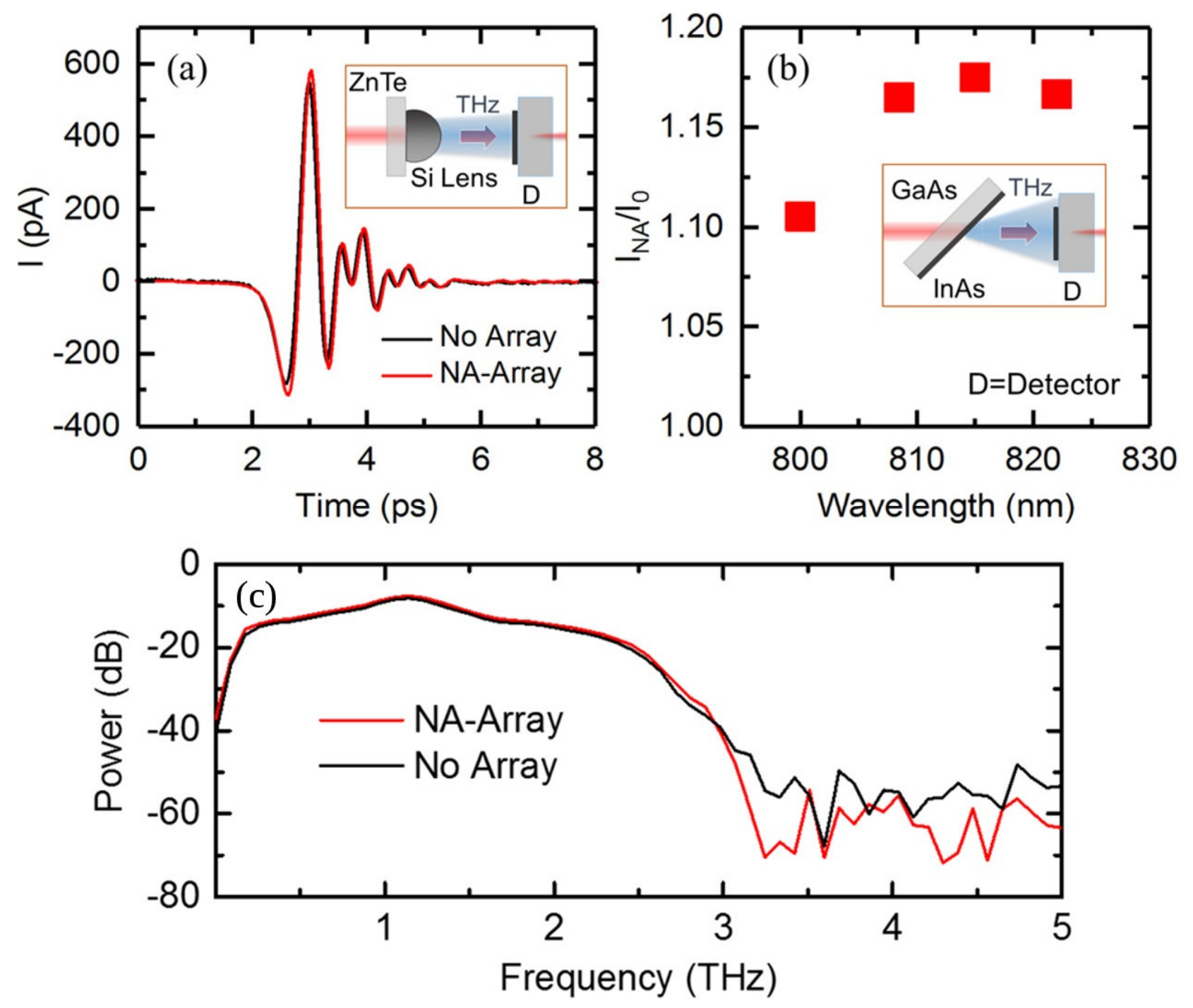

Figure 6. THz characterization of the hybrid cavity design. (a) The THz pulse detected with the nanoantenna array (red) and without (black). (b) Peak THz field detected with the hybrid cavity design, relative to the design without the nanoantenna array when the laser wavelength is varied. (c) Power spectrum of the waveforms shown in (a). ${ }^{12}$

\section{CONCLUSIONS}

We have analyzed the performance of the hybrid cavity design when three device parameters are varied: The thickness of the photoconductive layer, the periodicity of the nanoantenna array, and the individual nanoantenna length. We found the thickness of the photoconductive layer was of critical importance, as the hybrid cavity mode is only supported when $L_{\text {GaAs }}=50,160$ and $270 \mathrm{~nm}$. While the periodicity does not significantly affect the device performance, the length of the individual nanoantennas does. This must be chosen such that light reflected by the nanoantennas destructively interferes with light reflected by the DBR. THz characterization shows the nanoantennas do improve device performance at the operation wavelength $(\sim 815 \mathrm{~nm})$. 


\section{REFERENCES}

[1] Berry, C. W., Wang, N., Hashemi, M. R., Unlu, M. and Jarrahi, M., "Significant performance enhancement in photoconductive terahertz optoelectronics by incorporating plasmonic contact electrodes.," Nat. Commun. 4, 1622 (2013).

[2] Berry, C. W., Hashemi, M. R. and Jarrahi, M., "Generation of high power pulsed terahertz radiation using a plasmonic photoconductive emitter array with logarithmic spiral antennas,” Appl. Phys. Lett. 104(8), 81122 (2014).

[3] Heshmat, B., Pahlevaninezhad, H., Pang, Y., Masnadi-Shirazi, M., Burton Lewis, R., Tiedje, T., Gordon, R. and Darcie, T. E., "Nanoplasmonic terahertz photoconductive switch on GaAs," Nano Lett. 12(12), 6255-6259 (2012).

[4] Matsuura, S., Tani, M. and Sakai, K., "Generation of coherent terahertz radiation by photomixing in dipole photoconductive antennas," Appl. Phys. Lett. 70(5), 559 (1997).

[5] Moon, K., Lee, I.-M., Shin, J.-H., Lee, E. S., Kim, N., Lee, W.-H., Ko, H., Han, S.-P. and Park, K. H., "Bias field tailored plasmonic nano-electrode for high-power terahertz photonic devices.," Sci. Rep. 5, 13817 (2015).

[6] Wu, Q. Y. S., Tanoto, H., Ding, L., Choy Chum, C., Wang, B., Bian Chew, A., Banas, A., Banas, K., Jin Chua, S. and Teng, J., "Branchlike nano-electrodes for enhanced terahertz emission in photomixers.," Nanotechnology 26(25), 255201 (2015).

[7] Briscoe, J. L. and Cho, S.-Y., "Hybridised extraordinary optical transmission in plasmonic cavity," Electron. Lett. 50(24), 1860-1862 (2014).

[8] Atwater, H. a and Polman, A., "Plasmonics for improved photovoltaic devices.," Nat. Mater. 9(3), 205-213 (2010).

[9] Kim, S. J., Thomann, I., Park, J., Kang, J. H., Vasudev, A. P. and Brongersma, M. L., "Light trapping for solar fuel generation with Mie resonances," Nano Lett. 14(3), 1446-1452 (2014).

[10] Simovski, C., Morits, D., Voroshilov, P., Guzhva, M., Belov, P. and Kivshar, Y., "Enhanced efficiency of lighttrapping nanoantenna arrays for thin-film solar cells.," Opt. Express 21 Suppl 4(104), A714-25 (2013).

[11] Mitrofanov, O., Brener, I., Luk, T. and Reno, J. L., "Photoconductive Terahertz Near-Field Detector with a Hybrid Nanoantenna Array Cavity," ACS Photonics 2(12), 1763-1768 (2015).

[12] Thompson, R. J., Siday, T., Glass, S., Luk, T. S., Reno, J. L., Brener, I. and Mitrofanov, O., "Optically thin hybrid cavity for terahertz photo-conductive detectors," Appl. Phys. Lett. 110(4), 41105 (2017).

[13] Lumerical Solutions Inc., http://www.lumerical.com/tcad-products/fdtd/

[14] Munday, J. N. and Atwater, H. A., "Large Integrated Absorption Enhancement in Plasmonic Solar Cells by Combining Metallic Gratings and Antireflection Coatings," Nano Lett. 11(6), 2195-2201 (2011).

[15] Balanis, C. A., Antenna Theory: Analysis and Design, John Wiley \& Sons (2016). 